Article

\title{
Schematic Diagrams Design of Displacement Suppression Mechanism with One Degree-of-Freedom in a Rope-Guided Hoisting System
}

\author{
Lu Yan ${ }^{1,2}$, Guohua Cao ${ }^{1,2, *}$, Naige Wang ${ }^{1,2}$ and Weihong Peng ${ }^{3}$ \\ 1 School of Mechatronic Engineering, China University of Mining and Technology, Xuzhou 221116, China; \\ yanlu_088@cumt.edu.cn (L.Y.); wangng1990@foxmail.com (N.W.) \\ 2 Jiangsu Key Laboratory of Mine Mechanical and Electrical Equipment, China University of Mining and \\ Technology, Xuzhou 221116, China \\ 3 School of Mechanics and Civil Engineering, China University of Mining and Technology, Xuzhou 221116, \\ China; pengweihong@cumt.edu.cn \\ * Correspondence: caoguohua@cumt.edu.cn
}

Received: 16 January 2020; Accepted: 19 February 2020; Published: 18 March 2020

check for updates

\begin{abstract}
Since it is difficult for lateral stiffness of rope-guided rails to meet industry criteria in deep construction shaft, schematic diagrams of displacement suppression mechanisms (DSMs) are designed with a systematic approach demonstrated to reduce the lateral displacement of rope-guided rails in this paper. DSMs are simplified as planar four-bar and six-bar topological graphs based on topological theory. Each corresponding mechanical chain of these four-bar and six-bar mechanisms is divided into a rack, mechanical parts, prismatic, and revolute joints. An extended adjacency matrix is defined to represent the rack position, specific types of kinematic joints, and adjacency relationships between kinematic parts. Then, a symmetric vertex identification method is proposed with regard to planar 1-DOF (one degree of freedom) four-bar and six-bar topological graphs to get the sequences of prismatic joints for kinematic chains of DSMs. Finally, the alternative schematic diagrams of DSMs are obtained. The results show four-bar mechanisms with simple structure; few kinematical parts but less resident force are suitable for a mine shaft with small space and small swing. Six-bar mechanisms with two prismatic joints and three mechanical rack degree are applicable for wide shaft space in deep shaft, due to their stable structure and double resistant force. This development is helpful for DSM dimension synthesis design in future.
\end{abstract}

Keywords: rope-guided rail; displacement suppression mechanisms; vertex symmetry identification; schematic diagrams design

\section{Introduction}

With the increasing shortage of global resources and energy, many countries in the world are developing and utilizing mineral resources deeper in the earth. The excavation depth of newly-built mines in China has increased at an average speed of $10 \mathrm{~m}$ per year and mine hoisting in deep shaft requires higher speed, heavier load, and more stability. Wire ropes are widely used for the guidance of hosting conveyances in the process of shaft construction or in a mine hoisting system. Increasing depth of mine shaft leads to more insufficient stiffness of guiding ropes, thereby causing the substantial swinging of hoisting conveyances and increasing insecurity. Researchers made many studies about the stiffness $[1,2]$ of guiding ropes and the vibration $[3,4]$ in a rope-guided mine hoisting system. Therefore, the lateral deflection of conveyance and guiding ropes catch more attention in rope-guided hoisting 
systems. As shown in Figure 1, four suspension ropes, of which two ropes are utilized as guide rails, are tensioned at the end by gravity of platform. Since it is difficult to increase the lateral stiffness of rope guides only by enhancing the rope tension, displacement suppression mechanism (DSM) [5] was proposed in Figure 2 to achieve displacement suppression by a mechanical part swinging within a certain angle. DSM in Ref. [5] is a planar 1-DOF six-bar mechanism in topological structure. However, there are more mechanisms that can meet this condition according to synthesis theory of kinematic chains [6]. Thus, we need to design a proper mechanism used in limited mine shaft space. Linkage mechanism synthesis includes type synthesis and dimension synthesis. The former describes the number of mechanical parts and mechanical joins, as well as their adjacency relations, while the latter describes mechanism design and optimization of a certain kinematic chain. Nevertheless, the linkage mechanism type synthesis aims at topologies. Until now, many typical methods, like the characteristic polynomial method [7], Hamming number approach [8], ant algorithm [9], neural network algorithm [10], and genetic algorithm [11], have been used to solve isomorphism identification problem in topologies. However, there still exist many defectives in these methods, such as computation time, the number limitation of kinematic chains, and necessity and sufficiency. To solve the isomorphism problem with high efficiency, Xiao [12] constructed maximum structural code and then solved the traveling salesman problem based on ant and artificial immune system algorithms. Galán-Marín [13] solved a graph isomorphism problem with 28 vertices in seconds by using a new multivalued neural network method. Chang [14] compared the eigenvalue and eigenvector of adjacent matrix to find out the isomorphic relations between kinematic chains, and Cubillo [15] pointed out the non-sufficiency of Chang's method and revised the theory. Kuo [16] applied Rao's pseudo genetic algorithm [11] to identity vertex symmetry by comparing the vertex family strings under two conditions, i.e., internal topology adjacency and joint incidence. Later, Rao [17] defined a basic loop and an auxiliary structure to account the joints number between two basic vertices on basic loop or loose vertices on auxiliary structure by matrixes, which did not require the test for isomorphism. Huber [18] took the isomorphism test for line graphs with high symmetry or regularity. Zeng [19] proposed Dividing and Matching Algorithm and Yang [20] used incident matrices for isomorphism identification. Thus, the topological types and the corresponding pattern library were built gradually [21-23] and then topological graphs or mechanical chains were latter obtained by computer programs sketching automatically $[24,25]$. Rai [26] attempted to detect the isomorphism among the kinematic chains by calculate power and efficiency. Deng [27] adopted the idea of molecular topological index in organic chemistry isomer identification to obtain extended adjacency identification index, due to the similarity between chemical molecular model and topological graph of kinematic chain.

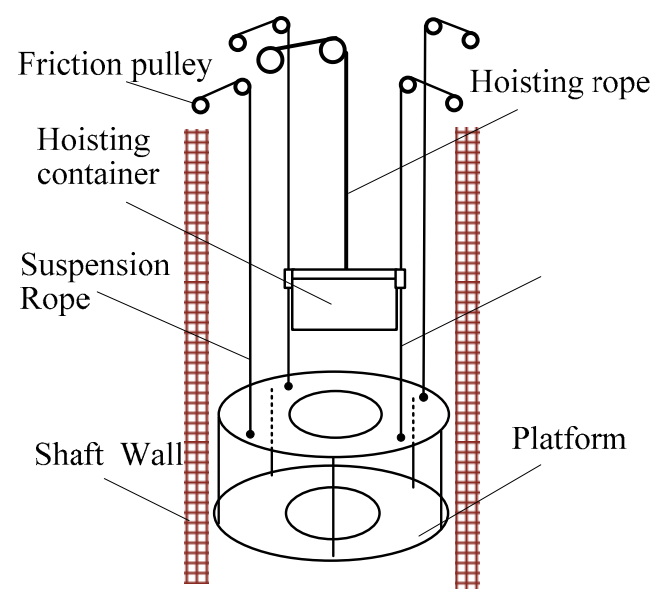

Figure 1. Construction shaft. 


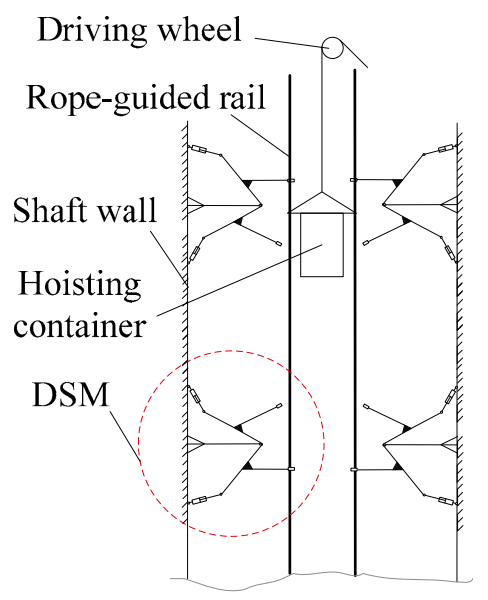

Figure 2. Displacement suppression scheme with rope-guided rail.

However, different from topological graph, the schematic diagram of mechanism includes different joint types and mechanism rack types. Since the generation from a topological graph or a kinematic chain to a schematic diagram does not make a one-to-one correspondence, due to symmetrical vertices and edges in topological graphs, i.e., several schematic diagrams may be obtained by one topological graph or kinematic chain. Therefore, Rao [28] used values of 2 and 1 in the link-link adjacency matrix to represent prismatic and revolute joints, respectively. Thus, schematic diagram isomorphism identification is less defined and concerned. Eleashy [29] obtained all solutions of planar 8-bar kinematic chains with up to three prismatic pairs by a systematic method. Dharanipragada [30] thoroughly considered the type difference between prismatic and revolute joints and studied the isomorphism for 1-DOF six-bar and an eight-bar mechanism by splitting Hamming String. However, it is worth noting that the existence of a mechanism rack may also lead to different schematic diagrams. Thus, it is necessary to identify the isomorphic schematic diagrams and obtain all these schematic diagrams of DSM. Finally, these mechanisms can be designed according to their working condition requirements, which are:

(1) One of the mechanical parts of DSM is capable of swinging within a certain angle.

(2) The mechanism can be locked at the limited position.

(3) It is a 1-DOF planar structure in order to implement easily.

Vertex symmetry in schematic diagram is different from that in a topology graph due to the distinct type among mechanical parts, such as rack and binary bar. Sun [31] proposed to improve Hamming number isomorphism determination based on a link-link adjacency matrix. This matrix contains the topological structure information of the kinematic chain. In order to obtain the required schematic diagrams of DSM, a new method is proposed to identity the schematic diagram isomorphism based on the unique nature of each kinematic chain. According to the definition of adjacency matrix, extended adjacency matrix is defined in this paper, which describes the certain type of kinematic joints, adjacency relations between two kinematic parts, and two mechanical rack positions. Then, the isomorphism identification procedure for schematic diagrams of DSM are derived based on four-bar and six-bar mechanisms. Sequences of prismatic joints for DSMs based on canonical perimeter are determined, and the alternative schemes for DSMs are obtained. This provides a new method for type synthesis of schematic diagram and also offers alternative models for the design of DSM.

The remainder of this paper is organized as follows. In Section 2, possible topological graphs for DSMs is presented and corresponding types of kinematic joints are enumerated. In Section 3 , connected relations of graphs are defined by two kinds of matrices to identify different topological graphs in Section 3.1 and corresponding schematic diagrams in Section 3.2, respectively. In addition, a symmetry discrimination method by two steps is proposed to identify whether any two vertices (or any two schematic diagrams) are symmetric in Section 3.3. In Section 4, detailed computational 
isomorphic discrimination steps are presented, and all the non-isomorphic vertexes, racks, as well as schematic diagrams of DSMs, are obtained by special types of sequence numbers. In Section 5, alternative schematic diagrams of DSM are screened and analyzed for further use in a rope-guided mine shaft. In Section 6, concluding remarks are presented.

\section{Topology Design for DSM}

According to the three working condition requirements of DSM, the number of its kinematic joints $P_{h}$ can be derived as

$$
P_{h}=\frac{3(n-1)-F}{2}
$$

where $n$ is the total number of topological vertexes (mechanical bars). $F$ denotes the number of DOFs and $F=1$. It is obvious that $n$ is an even number and $n \geq 4$ for the reason that $P_{h}$ and $n$ must be positive integers. Thus, $n=4,6,8,10$, etc. In this case, let $n=4$ or $n=6$ to simplify the mechanical structure, that is, four-bar and six-bar linkage mechanisms are chosen for schematic diagram design.

Topological graph and the corresponding kinematic chain of planar 1-DOF four-bar and six-bar mechanisms are shown in Figure $3 a, b$, Figure $4 a, b$ and Figure $5 a, b$, respectively. According to canonical perimeter loop theory in Ref. [21], the loop number " $r$ " of four-bar and six-bar mechanism are $r=4$ and $r=5,6$, respectively. Moreover, the way of labelling for topological graphs is determined based on this theory as shown in Figures 3-5.

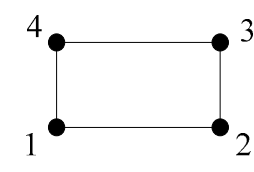

(a) Topological graph

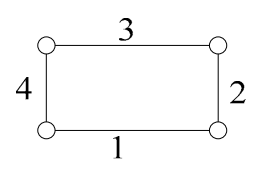

(b) Mechanic chain

Figure 3. Four-bar mechanism.

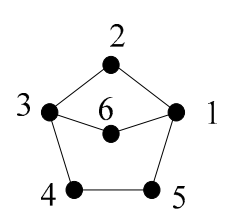

(a) Topological graph

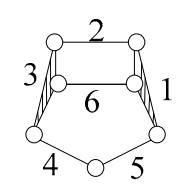

(b) Mechanic chain

Figure 4. Six-bar mechanism with the perimeter loop number of five $(r=5)$

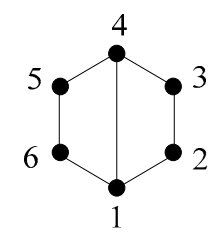

(a) Topological graph

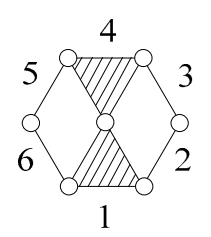

(b) Mechanic chain

Figure 5. Six-bar mechanism with the perimeter loop number of six $(r=6)$

Kinematic joints of DSM include prismatic joint $\mathrm{P}$ and revolute joint $\mathrm{R}$ in order to meet the condition that the mechanism can swing within a certain angle and be locked at a limited position. As shown in Figure 6, prismatic joint $P$ consists of a piston and a cylinder, and revolute joint $R$ is formed with any two of binary bars, pistons, and cylinders. A spring is used between the prismatic joint to ensure the DSMs locking in a limited position. 


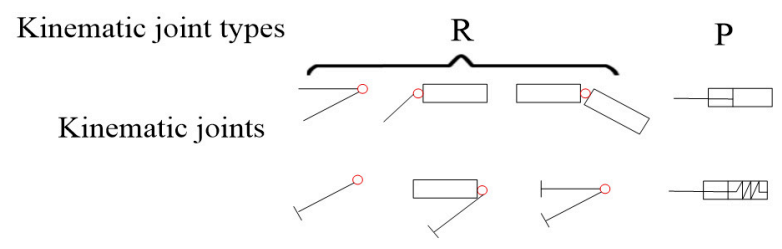

Figure 6. Kinematic joint types.

In order to describe the adjacent relations between kinematic parts, each kinematic part is presented with detailed kinematic joints and "RR, RP" and "RRR, RRP, RPP, PPP" are respectively used to represent different types of kinematic joints. "RR" denotes the kinematic part, which is connected to another two parts by two revolute joints. "RP" means that the kinematic part connected to another two bars is respectively by a revolute joint $R$ and a prismatic joint $P$. Similarly, "RRR", "RRP", "RPP", and "PPP" are the kinematic parts connected to another three parts by three R joints, two R joints, a P joint, an R joint, and two P joints, as well as three $\mathrm{P}$ joints, respectively. Since the prismatic joint is constituted by a cylinder block and a piston, there are several types of kinematic parts corresponding to "RP", "RRP", "RPP", and "PPP". All detailed kinematic parts in schematic diagrams of DSMs are shown in Figure 7. Mechanism racks are connected with these kinematic parts; hence, their structures include two types as shown in Figure 8a,b, respectively.

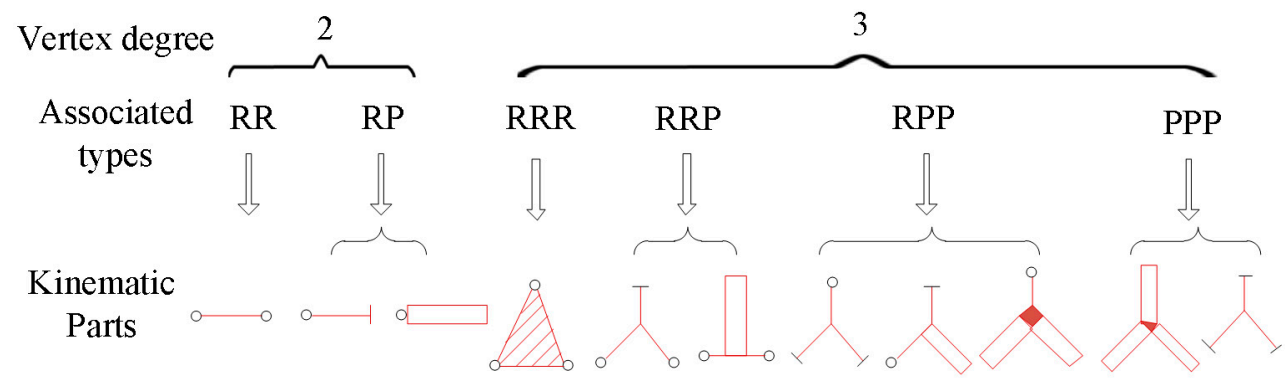

Figure 7. Kinematic part types.

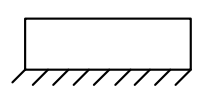

(a) Rack with prismatic joint

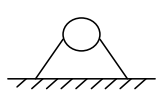

(b) Rack with revolute joint

Figure 8. Mechanical racks.

\section{Connected Relations of Graphs}

\subsection{Adjacency Relation of Topological Graph}

$G(V, E)$ is a topological graph where $V$ and $E$ are respectively defined as mathematical aggregations of vertexes and edges in Ref. [21]. According to the adjacent relationship between vertexes, adjacency matrix $A M(G)$ of topological graph $G$ is defined as:

$$
A M(G)=\left[(a m)_{i j}\right]_{n \times n}
$$

where $n$ is the vertex number of topological graph $G$ and

$$
a m_{i j}=\left\{\begin{array}{l}
1, \text { if vertexes } i \text { and } j \text { are connected by one edge } \\
0, \text { else }
\end{array}\right.
$$

\subsection{Adjacency Relation of Mechanism Diagram}

Adjacency matrix represents the adjacent relationships of vertexes in a topological graph; however, in the corresponding schematic diagram of mechanism, it can neither express the position of mechanical 
racks nor describe the type of kinematic joints. As with the definition of $G(V, E), M(R, C, J)$ is defined as a schematic diagram of mechanism where $R, C$, and $J$ denote the mathematical aggregations of mechanical racks, kinematic parts and kinematic joints, respectively. Therefore, $N(V)=N(R)+N(C)$ and $N(E)=N(J)$ when $N(X)$ is defined to represent the element number of mathematical aggregation $X$. Since one adjacency matrix of a topological graph corresponds to several schematic diagrams of mechanism, an extended adjacency matrix $\operatorname{EAM}(M)$ is defined as follows to accurately describe the position of mechanical rack and the joint type and $f$ is defined as any real number other than 0 and \pm 1 to represent mechanical rack:

$$
\operatorname{EAM}(M)=\left[(\text { eam })_{i j}\right]_{n \times n}
$$

where $n=N(V)$ and

$$
\operatorname{eam}_{i j}=\left\{\begin{array}{l}
-1, i \text { and } j \text { are connected by prismatic joint } \\
1, i \text { and } j \text { are connected by revolute joint } \\
f, i=j \text { and } i \text { represents mechanical rack } \\
0, \text { otherwise }
\end{array}\right.
$$

For example, both four-bar mechanisms, with a mechanical rack of label 1 in Figure 9, are developed from the topological graph in Figure $3 \mathrm{a}$ and include a prismatic joint and three revolute joints. Their adjacency matrix $A M(\mathrm{~F} 3)$ of Figure 3 and corresponding extended adjacency matrix are $E A M(\mathrm{~F} 9 \mathrm{a})$ of Figure $9 \mathrm{a}$ and $E A M(\mathrm{~F} 9 \mathrm{~b})$ of Figure $9 \mathrm{~b}$, respectively.

$$
A M(F 3)=\left[\begin{array}{llll}
0 & 1 & 0 & 1 \\
1 & 0 & 1 & 0 \\
0 & 1 & 0 & 1 \\
1 & 0 & 1 & 0
\end{array}\right], E A M(F 9 \mathrm{a})=\left[\begin{array}{cccc}
f & -1 & 0 & 1 \\
-1 & 0 & 1 & 0 \\
0 & 1 & 0 & 1 \\
1 & 0 & 1 & 0
\end{array}\right], E A M(F 9 \mathrm{~b})=\left[\begin{array}{cccc}
f & 1 & 0 & 1 \\
1 & 0 & -1 & 0 \\
0 & -1 & 0 & 1 \\
1 & 0 & 1 & 0
\end{array}\right]
$$
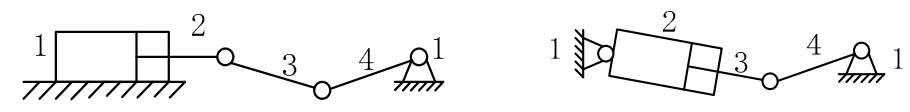

(a) Type A (b) Type B

Figure 9. Four-bar mechanism diagram.

\subsection{Vertexes Symmetry Identification}

Two symmetrical vertexes are the same in vertex degree and incident characteristics according to Kuo's symmetry identification [16]. Therefore, Kuo's method can identify vertexes with equal or equivalent adjacency symmetry. However, Kuo's method is complicated, due to generation matrix and cross matrix required to obtain fitness value and vertex family string. In addition, Kuo's method cannot be used for schematic diagram symmetry identification. Since a topological graph with its vertexes numbered corresponds to a certain adjacency matrix, adjacency matrixes of a topological graph with vertexes numbered by different methods can be applied to identify whether two vertexes are symmetric or equivalent. Liu [32] pointed out that, if there is a matrix $T$ making $T A T^{\mathrm{T}}=B$, where $A$ and $B$ are two adjacent matrixes of topological graphs, then two topological graphs are isomorphic. This method can be also used for schematic diagram symmetry identification.

Two types of symmetric vertexes exist in Figures $3 a$, $4 a$ and $5 a$, that is, equal adjacency symmetry and equivalent adjacency symmetry. Taking Figure $4 \mathrm{a}$ for example, vertexes 2 and 6 are equal adjacency symmetry because they are both connected with vertexes 1 and 3 , while vertexes 1 and 3 are equivalent adjacency symmetry, and they are symmetrical in topological structure; even if vertex 1 is connected with vertexes 2 and 5 , vertex 3 is connected with vertexes 2 and 4 . Therefore, two steps of symmetry discrimination method are proposed to identify whether any two vertices are symmetric. Detailed explanations are as Step (I) and Step (II): 
Step (I)—Equal adjacency symmetry: Two vertexes with equal adjacency symmetry exactly as vertexes 2 and 6 in Figure 4a have the same adjacent relationship with other vertexes, i.e., their adjacency matrixes are the same. Therefore, if two vertexes are equal adjacency symmetry, the corresponding row and column of a vertex in adjacent matrix are respectively equal to those of the other. Thus, transformation matrix TM $(p, q)$ is defined as Equation (4) to identify whether two vertexes in a topological graph have equal adjacency symmetry, where $p$ and $q$ are the label number of two vertexes:

$$
T M(p, q)=\left[(t m)_{i j}\right]_{n \times n^{\prime}} p=1,2, \ldots, n-1 \text { and } p<q
$$

where

$$
t m_{i j}=\left\{\begin{array}{l}
1, \text { if }(i=j \text { and } i \neq p, q) \text { or }(i=p, j=q) \text { or }(i=p, j=q) \\
0, \text { else }
\end{array}\right.
$$

In addition, the relationship between the new adjacency matrix $A M(j)$ and the original adjacency matrix $A M(G)$ is derived as:

$$
A M(j)=T M(p, q) \times A M(G) \times T M^{T}(p, q), j=(p-1) n-p(p-1) / 2+q-p
$$

where superscript $\mathrm{T}$ denotes a transpose of the matrix, and $j$ represents the graph number in each identification step. If $A M(j)=A M(G)$, vertexes $p$ and $q$ have equal adjacency symmetry. Taking transformation matrixes $T M(2,6)$ and $T M(1,3)$, for example, their expressions are:

$$
T M(2,6)=\left[\begin{array}{llllll}
1 & 0 & 0 & 0 & 0 & 0 \\
0 & 0 & 0 & 0 & 0 & 1 \\
0 & 0 & 1 & 0 & 0 & 0 \\
0 & 0 & 0 & 1 & 0 & 0 \\
0 & 0 & 0 & 0 & 1 & 0 \\
0 & 1 & 0 & 0 & 0 & 0
\end{array}\right] T M(1,3)=\left[\begin{array}{llllll}
0 & 0 & 1 & 0 & 0 & 0 \\
0 & 1 & 0 & 0 & 0 & 0 \\
1 & 0 & 0 & 0 & 0 & 0 \\
0 & 0 & 0 & 1 & 0 & 0 \\
0 & 0 & 0 & 0 & 1 & 0 \\
0 & 0 & 0 & 0 & 0 & 1
\end{array}\right]
$$

According to Equation (5) and Figure 4, $A M(9)=T M(2,6) \times A M(F 4) \times T M^{T}(2,6), A M(2)=T M$ $(1,3) \times A M(\mathrm{~F} 4) \times T M^{\mathrm{T}}(1,3)$ :

$$
A M(\mathrm{~F} 4)=\left[\begin{array}{cccccc}
0 & 1 & 0 & 0 & 1 & 1 \\
1 & 0 & 1 & 0 & 0 & 0 \\
0 & 1 & 0 & 1 & 0 & 1 \\
0 & 0 & 1 & 0 & 1 & 0 \\
1 & 0 & 0 & 1 & 0 & 0 \\
1 & 0 & 1 & 0 & 0 & 0
\end{array}\right] A M(9)=\left[\begin{array}{llllll}
0 & 1 & 0 & 0 & 1 & 1 \\
1 & 0 & 1 & 0 & 0 & 0 \\
0 & 1 & 0 & 1 & 0 & 1 \\
0 & 0 & 1 & 0 & 1 & 0 \\
1 & 0 & 0 & 1 & 0 & 0 \\
1 & 0 & 1 & 0 & 0 & 0
\end{array}\right] A M(2)=\left[\begin{array}{llllll}
0 & 1 & 0 & 1 & 0 & 1 \\
1 & 0 & 1 & 0 & 0 & 0 \\
0 & 1 & 0 & 0 & 1 & 1 \\
1 & 0 & 0 & 0 & 1 & 0 \\
0 & 0 & 1 & 1 & 0 & 0 \\
1 & 0 & 1 & 0 & 0 & 0
\end{array}\right]
$$

Since $A M(9)=A M(\mathrm{~F} 4)$ and $A M(2) \neq A M(\mathrm{~F} 4)$, it can be concluded that vertexes 2 and 6 have equal adjacency symmetry, while vertexes 1 and 3 are not. Therefore, this identification method is to detect whether the vertexes connected with two objective vertexes are the same.

Step (II)—Equivalent adjacency symmetry: If the adjacency matrix of a topological graph with vertexes numbered by a method is the same as that of the graph with vertexes numbered by another way, it can be concluded that two vertexes, with the same number respectively obtained through the above two methods, are symmetrical. This symmetry identification includes equivalent and equal adjacency symmetry. New adjacency matrixes will be obtained by reversely numbering and then sequentially renumbering the vertices of a graph, which are originally numbered by canonical perimeter numbering defined in Ref. [21]. Figure 4a is a canonical perimeter topological graph for a six bar mechanism. In order to find the vertexes symmetrical to vertex 1, the process in Figure 10 is presented as an example to describe the detailed steps. Figure 10a is the process of sequential numbering, and Figure $10 \mathrm{~b}$ includes two processes of reversely and sequentially numbering. The adjacent matrix of each topological graph after every sequential numbering process is different from 
that of the original one labeled with red number in Figure 10a, which indicates that there is no vertex symmetrical with vertex 1 . However, the adjacent matrix of a topological graph highlighted in red after step (4) in Figure 10b is equal to that of the original, which shows that vertexes 1 and 3 are symmetrical. It is easy to detect that the result of this process is sufficient but not the necessary condition to prove that two vertexes are symmetrical. This will be a necessary condition when the following preconditions are satisfied simultaneously:

1) Topological graphs are numbered on the basis of the perimeter loop theory and two vertexes to be identified are both in the canonical perimeter loop.

2) There is only one branch path outside the canonical perimeter loop.

3) Topological vertexes in sub-chain are no more than one.

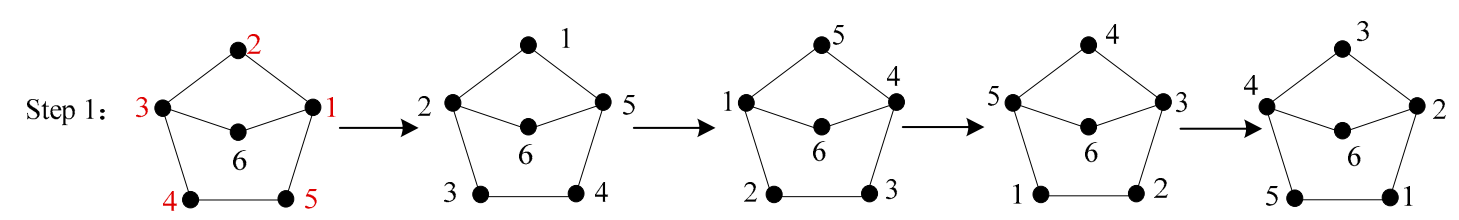

(a) Step 1: Sequential numbering in a counterclockwise direction

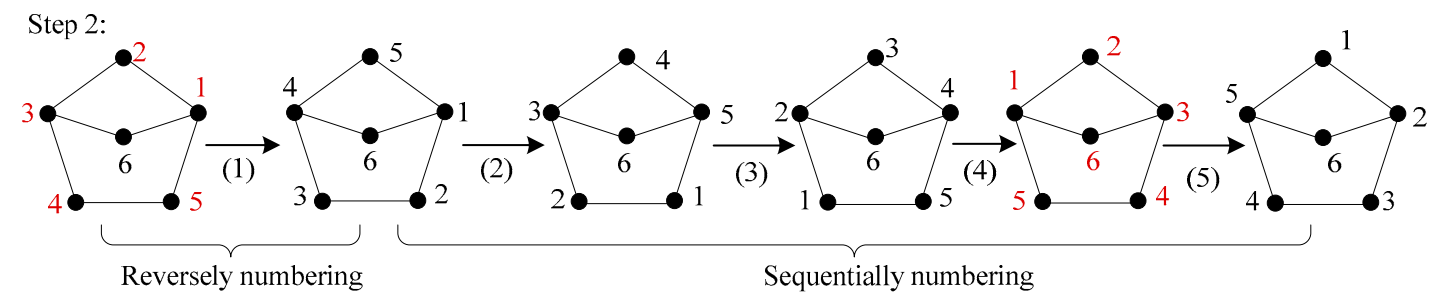

(b) Step 2: Sequentially numbering in clockwise direction

Figure 10. Transformation process.

In order to perform a traversal search on the perimeter loop with loop number $r$, the vertex numbered with $i$ in perimeter loop will be renumbered with $i-1(i=2,3, \ldots, r)$ or $r(i=1)$ in the sequential numbering process. The vertex numbered with $i$ in perimeter loop will be renumbered with $r+2-i(i=2,3, \ldots, r)$ or $1(i=1)$ in a reverse numbering process. The corresponding adjacency matrix generated from each step in Figure 10 can be described with transformation matrixes STM and RTM as Equations (6) and (7) to respectively express the steps of sequential numbering and reverse numbering:

$$
\begin{aligned}
& \text { STM }=\left[(\mathrm{stm})_{i j}\right]_{n \times n} \\
& \text { RTM }=\left[(\mathrm{rtm})_{i j}\right]_{n \times n}
\end{aligned}
$$

where

$$
\begin{aligned}
& r t m_{i j}=\left\{\begin{array}{l}
1, \text { if }\left[\left(\begin{array}{l}
i \\
j
\end{array}\right)=\left(\begin{array}{l}
1 \\
1
\end{array}\right),\left(\begin{array}{l}
r+1 \\
r+1
\end{array}\right),\left(\begin{array}{l}
r+2 \\
r+2
\end{array}\right) \ldots\left(\begin{array}{l}
n \\
n
\end{array}\right)\right] \text { or }\left[\left(\begin{array}{l}
i \\
j
\end{array}\right)=\left(\begin{array}{l}
2 \\
r
\end{array}\right),\left(\begin{array}{l}
3 \\
r-1
\end{array}\right) \ldots\left(\begin{array}{l}
r \\
2
\end{array}\right)\right] \\
0, \text { otherwise }
\end{array}\right. \\
& \operatorname{stm}_{i j}=\left\{\begin{array}{l}
1, \text { if }\left[\left(\begin{array}{l}
i \\
j
\end{array}\right)=\left(\begin{array}{l}
r+1 \\
r+1
\end{array}\right),\left(\begin{array}{l}
r+2 \\
r+2
\end{array}\right) \ldots\left(\begin{array}{l}
n \\
n
\end{array}\right)\right] \text { or }\left[\left(\begin{array}{l}
i \\
j
\end{array}\right)=\left(\begin{array}{l}
1 \\
2
\end{array}\right),\left(\begin{array}{l}
2 \\
3
\end{array}\right) \ldots\left(\begin{array}{l}
r-1 \\
r
\end{array}\right),\left(\begin{array}{l}
r \\
1
\end{array}\right)\right] \\
0, \text { otherwise }
\end{array}\right.
\end{aligned}
$$

The given adjacency matrix of a topological graph is set as $A M_{0}$ with its corresponding vertexes numbered in the counterclockwise direction. The new adjacency matrixes are set as $A M_{a}(i)$ and $A M_{b}(i)$ during the sequential numbering processes respectively in the counterclockwise and clockwise 
direction. Thus, the initial matrix $A M_{a}(1)$ and $A M_{b}(1)$ satisfy $A M_{a}(1)=A M_{b}(1)=A M_{0}$, thereby, $A M_{a}(i+1)$ and $A M_{b}(i+1)$ are derived as Equations (8) and (9), respectively:

$$
A M_{a}(i+1)=S T M \times A M_{a}(i) \times S T M^{\mathrm{T}}, i=1,2, \ldots, r-1
$$

The new adjacency matrix after each step is derived as:

$$
A M_{b}(i+1)=\left\{\begin{array}{l}
R T M \times A M_{b}(i) \times R T M^{\mathrm{T}}, i=1 \\
\operatorname{STM} \times A M_{b}(i) \times S T M^{\mathrm{T}}, i=2,3, \ldots, r
\end{array}\right.
$$

We can obtain that $A M_{b}(5)=A M_{b}$ (1) by computing each step in Figure 10 with Equations (8) and (9). The corresponding vertex numbers of $A M_{b}(5)$ are marked in red, which indicates that vertexes 1 and 3 are equivalent adjacency symmetry.

Four-bar and six-bar linkage mechanisms both fulfill conditions 2 and 3 in their topological structures. As for condition 1 , the equivalent adjacency symmetry method can identify two vertexes both in a canonical perimeter loop, while an equal adjacency symmetry method can identify two vertexes, respectively, in a sub-chain and canonical perimeter loop. Therefore, the two symmetry identifications above are workable. In addition, this symmetry identification is not only effective for topological graphs that satisfy the above conditions, but also feasible to identity symmetry for schematic diagram of mechanisms due to less concern about the process from a topological graph or mechanical chain to sundry schematic diagrams.

\section{Computational Identification}

Symmetry identification of mechanism diagrams is to distinguish the adjacent relations between sliding mechanical parts, revolute mechanical parts and their mechanical rack. In order to obtain all mechanism diagrams distinct in their kinematic joint types, we need to decide which vertex number is the mechanical rack firstly because two mechanism diagrams, with the same joint types and the same adjacent relation in topological graphs but two different label numbers of mechanical rack, may be two different structures. Then, the number of prismatic joints is the next step to be determined. The identification procedure to obtain an asymmetrical extended adjacent matrix is shown in Figure 11 and detailed steps and explanations are described as follows:

(i) Determine label number of mechanical rack: Two vertexes with topological symmetry form a vertex pair. All vertex pairs can be determined by symmetry identification of a topological graph. Among these symmetrical vertex pairs, the vertices between which have topological asymmetry, are chosen as labels of mechanical rack. The vertex labels of alternative rack are put into row matrix $a$ with the length of $N_{a}$.

(ii) Determine the number of prismatic joints: Since revolute joints are generally used in DSM and more prismatic joints will lead to more different mechanism diagram, one or two prismatic joints are both considered in the process to get mechanism diagrams. $N_{p}$ is used to denote the number of prismatic joints.

(iii) Obtain all possible extended adjacent matrixes: All extended adjacent matrixes of mechanism diagrams with one or two prismatic joints are put into the matrix RecordAM for the following symmetry identifications. Corresponding rack vertex label and the labels of vertexes connected by prismatic joints are stored into matrix LocP. [RecordAM_LocP] $=\operatorname{RestoreAM} \_$Loca $\left(A M_{0}, N_{p}, a\right.$, $n, r)$, where RestoreAM_Loca () is a user-defined MATLAB function.

(iv) Symmetry identification of mechanism diagrams: One extended adjacent matrix $E A M$ is taken out from RecordAM to compare with another extended adjacent matrix EAMx one by one. If $E A M \neq E A M x$, record $E A M$ in matrix $R_{x}$ and record the corresponding rack and prismatic joints information from $L o c P$ in matrix $L p_{x}$; otherwise, abnegate $E A M$ and identify the next one. $\left[R_{x} L P_{x}\right]$ 
$=$ SymmetryIdentP $\left(n, r, N_{p}, a, \operatorname{Record} A M, \operatorname{Loc} P\right)$, where SymmetryIdentP () is a user-defined MATLAB function.

(v) Discard the mechanism diagrams with two consecutive prismatic joints in a six-bar mechanism except those both connected with mechanism rack: Two consecutive prismatic joints mean a mechanical part is connected with other two parts both by prismatic joints, which leads to a more complex structure and more difficult diagram design relative to that in a four-bar mechanism. Since a mechanism rack is immovable, its position can be quickly determined according to required movement. The number of alternative mechanisms is $N_{P L x}=$ length $\left(L_{P x}\right) / N_{p}$, where length () is a MATLAB function to obtain the matrix length. If the common vertex of two consecutive prismatic joints is not equal to rack label, a corresponding extended adjacent matrix from matrix $R_{x}$ and a matrix of rack and prismatic joints information from $L p_{x}$ will be recorded in matrixes $f_{R}$ and $f_{L P}$, respectively.

(vi) Abandon six-bar mechanism diagrams with all prismatic joints in four-bar sub-loops and the mechanisms for which any prismatic joint exist in a sub-loop without a mechanism rack: From objective topological graphs, a sub-loop of a six-bar topological graph is four vertexes and will be substituted with four mechanical parts in a conversion process to the mechanism diagram. A six-bar mechanism with all prismatic joints existing in a four-bar sub-loop is almost equivalent to four-bar mechanisms, due to the following movement of the other sub-chain. In addition, if prismatic joints exist in this sub-loop without mechanism rack, there is little possibility to achieve locking in a limited position because the function is achieved by setting prismatic joints and a mechanism rack in the same sub-loop. Therefore, the above conditions can be neglected.

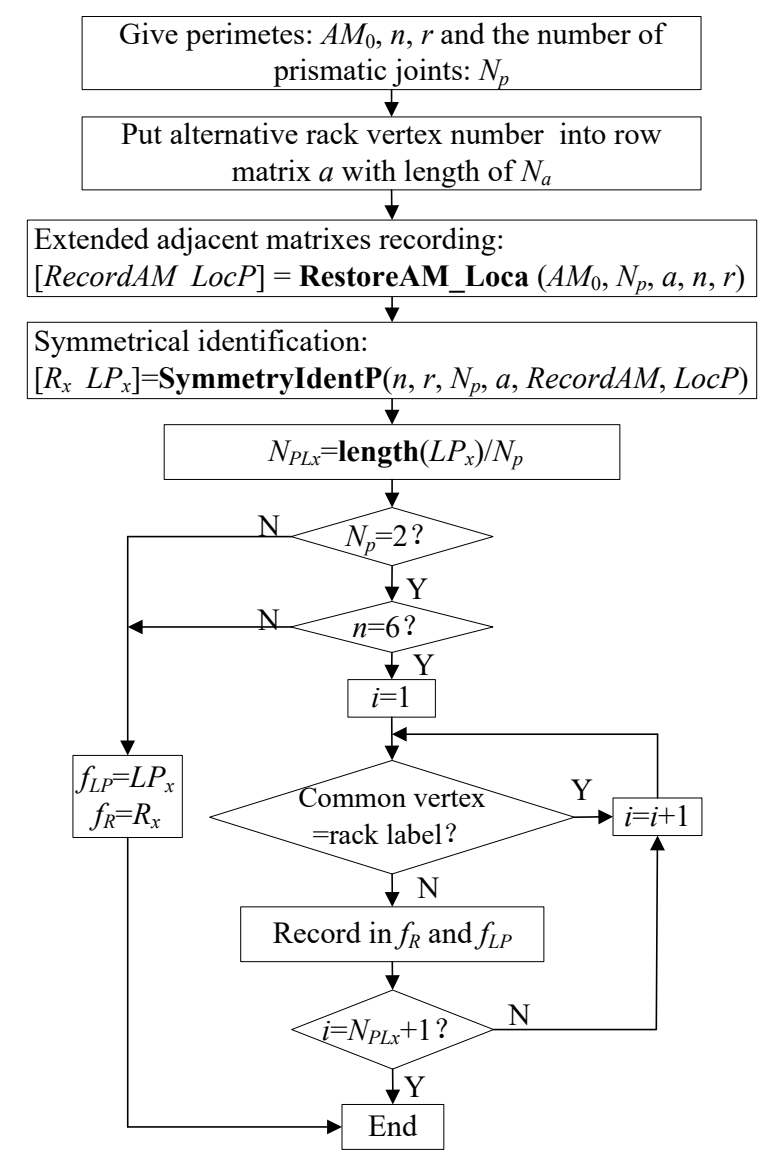

Figure 11. Identification procedure to obtain EAM. 
According to vertexes' symmetry identification in Section 2, the computational identification procedures from step $i$ to detect equal and equivalent symmetrical vertexes are demonstrated in Figure 12, where $R_{\mathrm{G}}$ with dimension of $n \times n$ is defined to display the information about two symmetrical vertexes and $R_{\mathrm{G}}(p, q)$ represent the element in the $p$ th row and the $q$ th column of $R_{\mathrm{G}}$. Thus, $R_{\mathrm{G}}(p, q)=1$ indicates that vertexes $p$ and $q$ have topological symmetry.

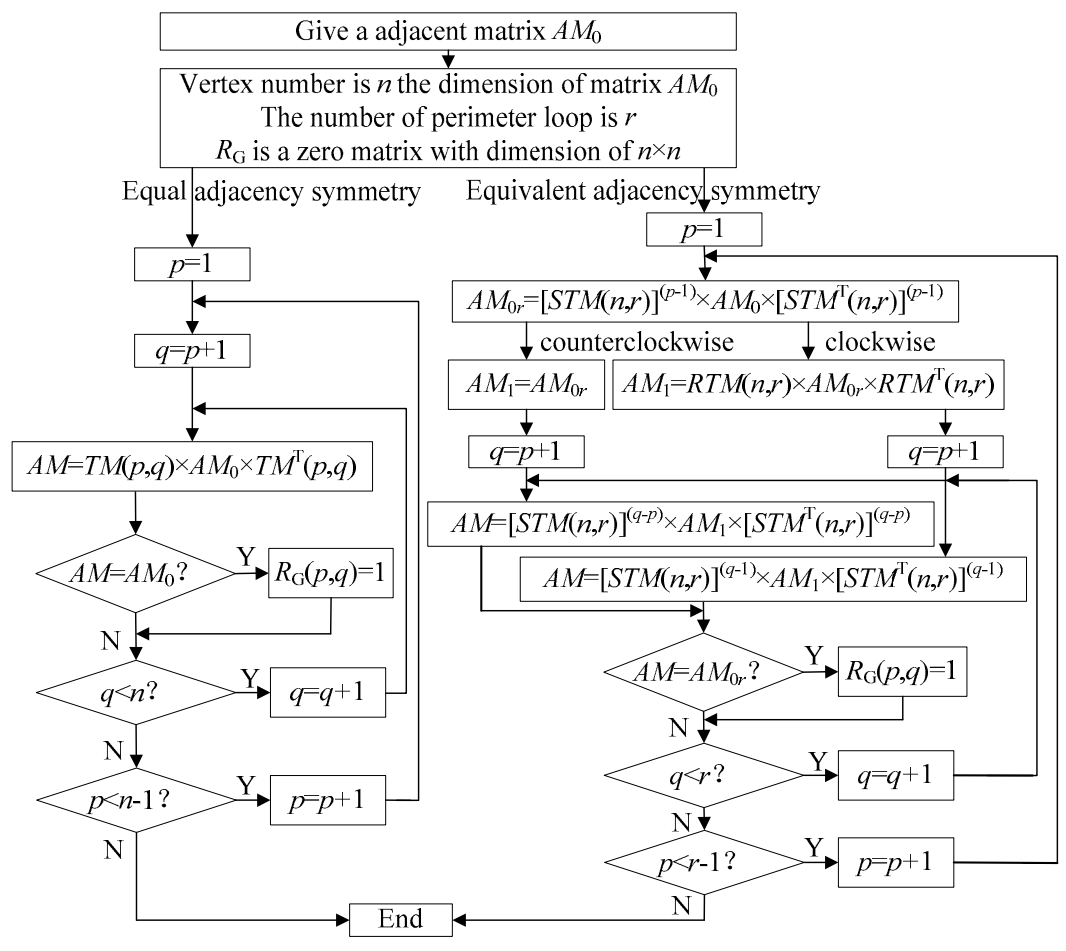

Figure 12. Symmetrical identification of vertexes.

$R_{\mathrm{G} 1}, R_{\mathrm{G} 2}$, and $R_{\mathrm{G} 3}$ calculated from Figure 11 are used to describe the topological symmetry of vertexes in Figures 3-5, where

$$
R_{1}=\left[\begin{array}{llll}
0 & 1 & 1 & 1 \\
0 & 0 & 1 & 1 \\
0 & 0 & 0 & 1 \\
0 & 0 & 0 & 0
\end{array}\right], R_{2}=\left[\begin{array}{llllll}
0 & 0 & 1 & 0 & 0 & 0 \\
0 & 0 & 0 & 0 & 0 & 1 \\
0 & 0 & 0 & 0 & 0 & 0 \\
0 & 0 & 0 & 0 & 1 & 0 \\
0 & 0 & 0 & 0 & 0 & 0 \\
0 & 0 & 0 & 0 & 0 & 0
\end{array}\right], R_{3}=\left[\begin{array}{llllll}
0 & 0 & 0 & 1 & 0 & 0 \\
0 & 0 & 1 & 0 & 1 & 1 \\
0 & 0 & 0 & 0 & 1 & 1 \\
0 & 0 & 0 & 0 & 0 & 0 \\
0 & 0 & 0 & 0 & 0 & 1 \\
0 & 0 & 0 & 0 & 0 & 0
\end{array}\right]
$$

$\mathrm{A}-\mathrm{B}$ is used to describe that vertexes $\mathrm{A}$ and $\mathrm{B}$ have topological symmetry. It can be easily concluded that three symmetrical vertex pairs $\mathrm{A}-\mathrm{B}, \mathrm{B}-\mathrm{C}$, and $\mathrm{A}-\mathrm{C}$ denote that $\mathrm{A}, \mathrm{B}$, and $\mathrm{C}$ are mutually symmetrical. Therefore, matrix $R_{1}$ denotes that vertex pairs with topological symmetry are 1-2, 1-3, 1-4, 2-3, 2-4, 3-4, and vertexes 1, 2, 3, 4 have mutual symmetry. According to this principle, symmetrical vertex pairs and the determined rack positions can be derived as shown in Table 1.

Table 1. Topological symmetry vertex pairs and rack labels.

\begin{tabular}{cccc}
\hline Topological Graph & Four Bar & Six Bar with $\boldsymbol{r}=\mathbf{5}$ & Six Bar with $\boldsymbol{r}=\mathbf{6}$ \\
\hline Symmetric vertex pairs & $\begin{array}{c}1 \sim 2,1 \sim 3,1 \sim 4,2 \sim 3,2 \sim 4, \\
3 \sim 4\end{array}$ & $2 \sim 6,1 \sim 3,4 \sim 5$ & $\begin{array}{c}1 \sim 4,2 \sim 3,2 \sim 5,2 \sim 6,3 \sim 5, \\
3 \sim 6,5 \sim 6\end{array}$ \\
\hline Mechanism rack label & 1 & $1,2,4$ & 1,2 \\
\hline
\end{tabular}


According to link adjacency and joint incidence theory and the method in Ref. [16], if two vertex family strings of all their corresponding cross matrixes are the same, two vertexes are proved to be isomorphic. $\left\{F_{i}\right\}_{1-L}$ denotes the family string of the $i$ th vertex with $L$ generation matrixes in a topological graph.

The vertex family strings of four-bar mechanism shown in Figure 3a are:

$$
\left\{F_{1}\right\}_{1-2}=\left\{F_{2}\right\}_{1-2}=\left\{F_{3}\right\}_{1-2}=\left\{F_{4}\right\}_{1-2}=\left\{\begin{array}{l}
8-2(4) \\
6-3(2)
\end{array}\right\}
$$

Vertex family strings of six-bar mechanism with $r=5$ shown in Figure 4a are:

$$
\begin{gathered}
\left\{F_{1}\right\}_{1-2}=\left\{F_{3}\right\}_{1-2}=\left\{\begin{array}{l}
20-(2),(3), 3(5) \\
18-3(3),(4),(5)
\end{array}\right\} \\
\left\{F_{2}\right\}_{1-2}=\left\{F_{6}\right\}_{1-2}=\left\{\begin{array}{l}
14-2(2), 2(5) \\
16-(2), 2(3), 2(4)
\end{array}\right\}, \\
\left\{F_{4}\right\}_{1-2}=\left\{F_{5}\right\}_{1-2}=\left\{\begin{array}{l}
16-2(2),(3),(4),(5) \\
17-(2), 2(3),(4),(5)
\end{array}\right\}
\end{gathered}
$$

Vertex family strings of four-bar mechanism with $r=6$ shown in Figure 5 a are:

$$
\begin{gathered}
\left\{F_{1}\right\}_{1-3}=\left\{F_{4}\right\}_{1-3}=\left\{\begin{array}{l}
14-2(1), 2(5),(6) \\
16-2(2), 3(4) \\
4-4(1)
\end{array}\right\} \\
,\left\{F_{2}\right\}_{1-3}=\left\{F_{3}\right\}_{1-3}=\left\{F_{5}\right\}_{1-3}=\left\{F_{6}\right\}_{1-3}=\left\{\begin{array}{l}
14-(1),(2), 2(4),(5) \\
16-2(2), 3(4) \\
8-2(1), 3(2)
\end{array}\right\}
\end{gathered}
$$

The results calculated by the method in Ref. [16] show that four-bar mechanism family strings of vertexes 1-4 are the same, which represents that vertexes 1-4 in Figure 3 a are isomorphic or symmetric. Similarly, vertexes between 2 and 6 , vertexes between 1 and 3 , and vertexes between 4 and 5 in Figure $4 a$ are symmetric, respectively. In Figure 5, Vertexes 1 and 3 are symmetric, and vertexes 2, 3, 5, 6 are mutually symmetric. These results are consistent with that in Table 1 and indicate the validity of

\begin{tabular}{|c|c|c|c|c|c|c|c|c|c|c|c|c|}
\hline \multirow{3}{*}{$\begin{array}{c}\text { Topological Graph } \\
\text { Rack } \\
N_{p}\end{array}$} & \multirow{2}{*}{\multicolumn{2}{|c|}{$\begin{array}{c}\text { Four Bar } \\
1\end{array}$}} & \multicolumn{6}{|c|}{ Six Bar with $r=5$} & \multicolumn{4}{|c|}{ Six Bar with $r=6$} \\
\hline & & & \multicolumn{2}{|c|}{1} & \multicolumn{2}{|c|}{2} & \multicolumn{2}{|c|}{4} & \multicolumn{2}{|c|}{1} & \multicolumn{2}{|c|}{2} \\
\hline & 1 & 2 & 1 & 2 & 1 & 2 & 1 & 2 & 1 & 2 & 1 & 2 \\
\hline$N_{R}$ & 4 & 6 & 7 & 21 & 7 & 21 & 7 & 21 & 7 & 21 & 7 & 21 \\
\hline$N_{l p x}$ & 2 & 4 & 5 & 13 & 4 & 12 & 6 & 13 & 4 & 12 & 7 & 21 \\
\hline$N_{f}$ & 2 & 4 & 5 & 8 & 4 & 7 & 6 & 7 & 4 & 8 & 7 & 12 \\
\hline$N_{z}$ & 2 & 4 & 3 & 6 & 0 & 0 & 3 & 2 & 0 & 5 & 0 & 0 \\
\hline
\end{tabular}
symmetry identification of this paper.

According to the steps from (i) to (vi) and mechanism rack label in Table 1, the number of all mechanisms $N_{R}, N_{l p x}, N_{f}$ and $N_{z}$ can be obtained as Table 2, where $N_{R}, N_{l p x}$ and $N_{f}$ are calculated from matrixes $L o c P, L P_{x}$ and $f_{L P}, N_{z}$ is obtained from step (vi).

Table 2. Number of mechanisms from each step.

(A, B) is used to describe a pair of prismatic joint between vertexes $\mathrm{A}$ and $\mathrm{B}$. When $N_{p}=2$, two pairs of prismatic joint are separated with ".". Therefore, the final alternative mechanisms in Table 2 correspond to prismatic joints as shown in Table 3. 
Table 3. Sequence of prismatic joints.

\begin{tabular}{|c|c|c|c|c|c|c|c|}
\hline \multirow{2}{*}{\multicolumn{2}{|c|}{$\begin{array}{c}\text { Topological Graph } \\
\text { Rack }\end{array}$}} & \multirow{2}{*}{$\begin{array}{c}\text { Four Bar } \\
1\end{array}$} & \multicolumn{3}{|c|}{ Six Bar with $r=5$} & \multicolumn{2}{|c|}{ Six Bar with $r=6$} \\
\hline & & & 1 & 2 & 4 & 1 & 2 \\
\hline \multirow[b]{2}{*}{$\begin{array}{l}\text { Prismatic } \\
\text { joints }\end{array}$} & $N_{p}=1$ & $\begin{array}{l}(1,4) \\
(3,4)\end{array}$ & $\begin{array}{l}(1,5) \\
(3,4) \\
(4,5)\end{array}$ & & $\begin{array}{l}(1,5) \\
(3,4) \\
(4,5)\end{array}$ & & \\
\hline & $N_{p}=2$ & $\begin{array}{l}(1,2) \cdot(1,4) \\
(1,2) \cdot(2,3) \\
(1,4) \cdot(2,3) \\
(2,3) \cdot(3,4)\end{array}$ & $\begin{array}{l}(1,2) \cdot(3,4) \\
(1,2) \cdot(4,5) \\
(1,5) \cdot(1,6) \\
(1,5) \cdot(2,3) \\
(1,5) \cdot(3,4) \\
(2,3) \cdot(4,5)\end{array}$ & & $\begin{array}{l}(1,5) \cdot(3,4) \\
(3,4) \cdot(4,5)\end{array}$ & $\begin{array}{l}(1,2) \cdot(1,6) \\
(1,6) \cdot(2,3) \\
(1,6) \cdot(3,4) \\
(2,3) \cdot(5,6) \\
(3,4) \cdot(5,6)\end{array}$ & \\
\hline
\end{tabular}

\section{Alternatives for DSM and Results}

In order to describe these schematic diagrams numbered by the perimeter loop, a new description is proposed as their types of mechanisms. The sequence for each schematic diagram of mechanism is expressed as NB.NPL-RP-NP-PSP, where NB, NPL, RP, NP, and PSP denotes the number of bars, the number of perimeter loop, rack label, the number of prismatic joint, and the sequence of prismatic joints in Table 3, respectively. All schematic diagrams of DSM are shown in Figures 13-15. It can be seen that all schematic diagrams fulfill the previous motion requirements: swinging in a certain angle and being locked in two limited positions.
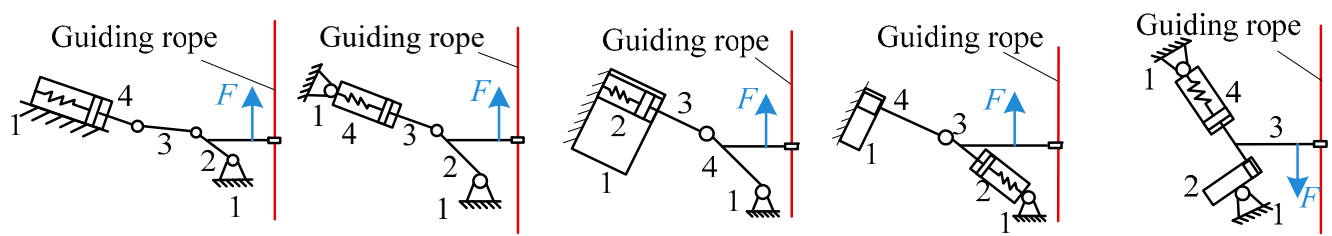

4.4-1-1- $(1,4)$ $4.4-1-1-(3,4)$

4.4-1-2-(1,2).(2,3)

4.4-1-2-(1,4). $(2,3)$

4.4-1-2-(2,3). $(3,4)$

Figure 13. Four-bar mechanism.
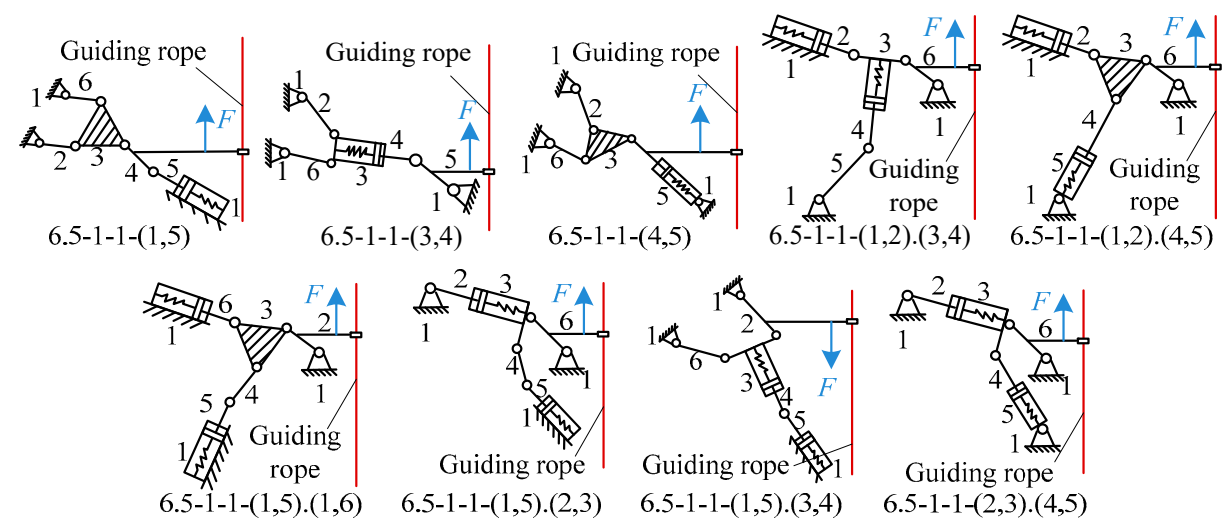

(a) Rack label is vertex 1
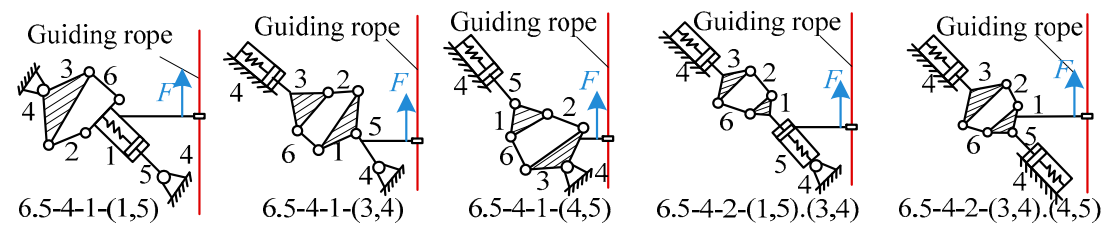

(b) Rack label is vertex 4

Figure 14. Six-bar mechanism with $r=5$. 


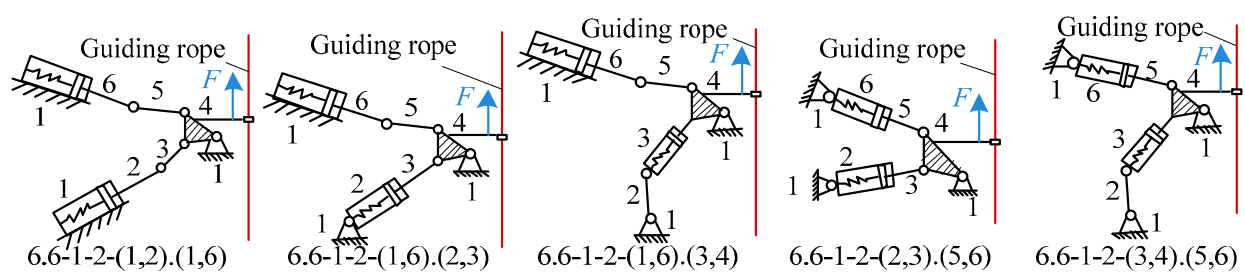

Figure 15. Six-bar mechanism with $r=6$.

Analyzing from the kinematic structure in Figure 13, a four-bar mechanism can be divided into two categories, respectively with one P-joint and two P-joints. Four-bar mechanisms with one P-joint are simple in structure and easy to design, while those with two P-joints need more elaborate design for the direction and position of initiative force, especially the bar as mechanical rack with a P-joint. Moreover, a mechanical rack with a P-joint in Figure 13 may have problems of insufficient locking force in their limited position because one of the prismatic joints without a spring only provides sliding motion and its motion direction is limited.

Comparing schematic diagrams in Figure 14 with that in Figure 15, six-bar mechanisms are more stable in structure when their rack degree is three, while they will lose this advantage when their rack degree is two. In general, six-bar mechanisms with two prismatic joints in Figures 14 and 15 are stable against resisting the rope displacement, but the failure rate will be higher than that of four-bar mechanism and six-bar mechanism with single P-joint. When $n=6, r=5$ and $N_{p}=1$ as shown in Figure 14a with single prismatic joint, since there are five minimum sub-loops with prismatic joints while the others have four, there are more mechanical bars to swing, and they need a great driving force. When $n=6, r=5$, and rack label is vertex 4 in Figure 14b, these types of mechanisms have a smaller swing angle, owing to a closed four-bar sub-loop in each of them. In this case, we can choose four-bar mechanisms with single prismatic joints for first priority in the shaft, and then the six-bar mechanism with two prismatic joints if more stable resistant forces are needed.

Based on the above analysis about Figures 13-15, we can find that the four-bar mechanisms are simple in structure, easy to design, have less resistance force, and occupy a small space compared with the six-bar mechanism. Thus, four-bar mechanisms are suitable for mine shafts with small space and small swing. Six-bar mechanisms with two prismatic joints and three mechanical rack degree are applicable for wide shaft space in deep shaft, due to their stable structure and double resistant force. This development is helpful for DSM dimension synthesis design in the future.

\section{Conclusions}

We determine possible topological graphs and the kinematic joints type of schematic diagrams, according to three working condition requirements of DSMs in this paper. Then, isomorphism identification by two steps of schematic diagrams is proposed, and the computational process is presented to obtain the alternative schematic diagrams of DSMs in rope-guided mine shaft.

Two concepts of equal symmetry and equivalent symmetry are proposed to identify the symmetrical vertexes and isomorphic schematic diagrams by changing the vertex label order. It is a simple and efficient way to obtain schematic diagrams of DSM, with 1-DOF four-bar and six-bar mechanisms, by two steps of symmetric identification in rope-guided mine shaft in three working conditions. Four-bar mechanisms with simple structure, few kinematical parts but less resident force are suitable for mine shafts with small space and small swing. Six-bar mechanisms with two prismatic joints and three mechanical rack degree are applicable for wide shaft space in deep shaft.

It is worth noting that alternative schematic diagrams for 4-bar and six-bar mechanisms are sufficient. Therefore, $8,10,12$, or more bar mechanisms are not taken into consideration, due to multiple bar mechanisms leading to the complexity for the required simple motion of DSM in mine shafts. In addition, each alternative schematic diagram of DSM presented in this paper can satisfy different mine shaft space, wideness, or straightness. No matter which structure we choose, further 
dimension synthesis to each structure is still required to be solved. Thus, it is important for further choice and design of DSM in rope-guided mine shaft.

Author Contributions: L.Y. and G.C. contributed equally to conceptualization and methodology of this paper. L.Y. and W.P.: references and literature collection and organization. L.Y. and N.W.: validation and main analysis, G.C; supervision, project administration and funding acquisition. All authors have read and agreed to publish this version of the paper.

Funding: This research received no external funding.

Acknowledgments: This work is supported by the Fundamental Research Funds for the Central Universities (2017XKQY038) and the Priority Academic Program Development of Jiangsu Higher Education Institutions (PAPD).

Conflicts of Interest: The authors declare no conflicts of interest.

\section{References}

1. Greenway, E.M. Lateral stiffness and deflection of vertical ropes with application to mine shaft hoisting. Aust. J. Mech. Eng. 2008, 5, 59-70. [CrossRef]

2. Yan, L.; Cao, G.; Wang, N.; Li, J. Lateral stiffness and deflection characteristics of guide cable with multi-boundary constraints. Adv. Mech. Eng. 2017, 9. [CrossRef]

3. Cao, G.; Wang, J.; Zhu, Z. Coupled vibrations of rope-guided hoisting system with tension difference between two guiding ropes. Proc. Inst. Mech. Eng. Part. C. J. Mech. Eng. Sci. 2018, 232, 231-244. [CrossRef]

4. Wang, J.; Pi, Y.; Hu, Y.; Gong, X. Modeling and dynamic behavior analysis of a coupled multi-cable double drum winding hoister with flexible guides. Mech. Mach. Theory 2017, 108, 191-208. [CrossRef]

5. Cao, G.; Wang, J.; Zhu, Z. Guide rail rope deflection inhibition mechanism and method for parallel soft cable suspension system. US Invention Patent US09689257B2, 22 January 2014.

6. Yan, H.; Chiu, Y. On the number synthesis of kinematic chains. Mech. Mach. Theory 2015, 89, 128-144. [CrossRef]

7. Uicker, J.J; Raicu, A. A Method for the Identification of and Recognition of Equivalence of Kinematic Chains. Mech. Mach. Theory 1975, 10, 375-383. [CrossRef]

8. Rao, A.C.; Varada, R.D. Application of the hamming number technique to detect isomorphism among kinematic chains and inversion. Mech. Mach. Theory 1991, 26, 55-75. [CrossRef]

9. Yang, P.; Pei, Z.H.; Liao, N.B.; Yang, B. Isomorphism identification for epicyclic gear mechanism based on mapping property and ant algorithm. Eng. Comput. 2007, 23, 49-54. [CrossRef]

10. Kong, F.G.; Li, Q.; Zhang, W.J. An artificial neural network approach to mechanism kinematic chain isomorphism identification. Mech. Mach. Theory 1999, 34, 271-283. [CrossRef]

11. Rao, A.C. A Genetic Algorithm for Topological Characteristics of Kinematic Chains. J. Mech. Des. 2000, 122, 228-231. [CrossRef]

12. Xiao, R.; Tao, Z.; Liu, Y. Isomorphism identification of kinematic chains using novel evolutionary approaches. Trans. ASME J. Comput. Inf. Sci. Eng. 2005, 5, 18-24. [CrossRef]

13. Galán-Marín, G.; López-Rodríguez, D.; Mérida-Casermeiro, E. A new multivalued neural network for isomorphism identification of kinematic chains. J. Comput. Inf. Sci. Eng. 2010, 10, 011009. [CrossRef]

14. Chang, Z.Y.; Zhang, C.; Yang, Y.H.; Wang, Y.X. A new method to mechanism kinematic chain isomorphism identification. Mech. Mach. Theory 2002, 37, 411-417. [CrossRef]

15. Cubillo, J.P.; Wan, J.B. Comments on mechanism kinematic chain isomorphism identification using adjacent matrices. Mech. Mach. Theory 2005, 40, 131-139. [CrossRef]

16. Kuo, C.H.; Shih, C.J. Computational identification of link adjacency and joint incidence in kinematic chains and mechanisms. J. Mech. Des. 2008, 130, 084501. [CrossRef]

17. Rao, A.C.; Deshmukh Pratap, B. Computer aided structural synthesis of planar kinematic chains obviating the test for isomorphism. Mech. Mach. Theory 2001, 36, 489-506. [CrossRef]

18. Huber, M. Computational complexity of reconstruction and isomorphism testing for designs and line graphs. J. Comb. Theory Ser. A 2011, 118, 341-349. [CrossRef]

19. Zeng, K.; Fan, X.; Dong, M.; Yang, P. A fast algorithm for kinematic chain isomorphism identification based on dividing and matching vertices. Mech. Mach. Theory 2014, 72, 25-38. [CrossRef] 
20. Yang, F.; Deng, Z.; Tao, J.; Li, L. A new method for isomorphism identification in topological graphs using incident matrices. Mech. Mach. Theory 2012, 49, 298-307. [CrossRef]

21. Ding, H.F.; Huang, Z. The establishment of the canonical perimeter topological graph of kinematic chains and isomorphism identification. J. Mech. Des. 2006, 129, 915-923. [CrossRef]

22. Ding, H.; Zhao, J.; Huang, Z. Unified structural synthesis of planar simple and multiple joint kinematic chains. Mech. Mach. Theory 2010, 45, 555-568. [CrossRef]

23. Ding, H.; Hou, F. Synthesis of the whole family of planar 1-DOF kinematic chains and creation of their atlas database. Mech. Mach. Theory 2012, 47, 1-15. [CrossRef]

24. Wu, X.H.; Nie, S.H.; Li, N. An approach of original loop to automatic sketching of planar kinematic chains. Mech. Sci. Technol. Aerosp. Eng. 2009, 28, 546-552.

25. Nie, S.H.; Liu, H. Maximal Loop Method of Automatic Sketching of Planar Closed Kinematic Chains. J. Mech. Eng. 2009, 45, 30-37. [CrossRef]

26. Rai, R.K.; Punjabi, S. Kinematic chains isomorphism identification using link connectivity number and entropy neglecting tolerance and clearance. Mech. Mach. Theory 2018, 123, 40-65. [CrossRef]

27. Deng, T.; Xu, H.; Tang, P.; Liu, P.; Yan, L. A novel algorithm for the isomorphism detection of various kinematic chains using topological index. Mech. Mach. Theory 2020, 146, 103740. [CrossRef]

28. Rao, A.C.; Nageswara Rao, C. Isomorphism among kinematic chains with Sliding Pairs. Indian J. Technol. $1989,27,363-365$.

29. Eleashy, H. A new atlas for 8-bar kinematic chains with up to 3 prismatic pairs using Joint Sorting Code. Mech. Mach. Theory 2018, 124, 118-132. [CrossRef]

30. Dharanipragada, V.; Chintada, M. Split hamming string as an isomorphism test for one degree-of-freedom planar simple-jointed kinematic chains containing sliders. J. Mech. Des. 2016, 138, 082301-082308. [CrossRef]

31. Sun, W.; Kong, J.Y.; Wang, X.D.; Hou, Y. Description and isomorphism judgment of the kinematic chain with multiple joints based on link-link adjacency matrix. J. Mech. Eng. 1-6. Available online: http: //kns.cnki.net/kcms/detail/11.2187.TH.20191224.1509.176.html (accessed on 25 December 2019).

32. Liu, H.; Shi, S.Y.; Yang, P.; Yang, J. An improved genetic algorithm approach on mechanism kinematic structure enumeration with intelligent manufacturing. J. Intell. Robot. Syst. 2018, 89, 343-350. [CrossRef]

(C) 2020 by the authors. Licensee MDPI, Basel, Switzerland. This article is an open access article distributed under the terms and conditions of the Creative Commons Attribution (CC BY) license (http://creativecommons.org/licenses/by/4.0/). 